\title{
Effects of bilateral lesions in the hippocampus on acquisition of two maze problems'
}

\author{
THERESA A. SPIEgEL, GAYLE HOSTETTER ${ }^{2}$ AND GARTH J. THOMAS ${ }^{3}$
}

UNIVERSITY OF ILLINOIS

Rats with hippocampal lesions and operated control rats were tested in an open field and trained on an alley maze and on an elevated maze (multiple-Ts). Deficits on both mazes occurred in the lesioned $S$ s with no significant difference between experimental and control groups in wall-following tendencies. Enhanced thigmotaxis cannot account for the deleterious effects of hippocampal lesions on maze learning. The lesions were relatively small (25\%) and did not invade the thalamus.

Several studies have shown that lesions in the hippocampus of rats severely impair learning and retention of mazes. Kimble (1963) reported that rats with lesions in the hippocampus made more errors on an easy pattern (1) of the Hebb-Williams maze test than they did on a more difficult pattern (6). Also, lesioned rats were reported to remain near the walls more than normal rats during an open-field test. These findings suggested to Hostetter \& Thomas (in press) that impairment in maze performance of rats with hippocampal lesions might stem from lesion-enhancement of the thigmotaxic tendency of normal rats. If this hypothesis were true, rats with lesions in the hippocampus should make more errors in an alley maze where an enhanced tendency to follow walls might lead them into blind alleys than they would on an elevated maze where there are no walls to follow. Hostetter \& Thomas (in press) found that the performance of hooded rats with dorsal hippocampal lesions was impaired on both mazes.

The present experiment extends the generality of these findings by demonstrating that bilateral lesions in the ventral hippocampus in a different strain of rats (albino Wistars) also severely impair maze performance, and that there is no interaction between the effect of the lesions and the type of maze. The results also show that small lesions in the hippocampus that do not bilaterally invade the optic system, nonetheless, impair performance.

Method

Subjects. Fourteen male albino rats (Wistar strain), each weighing approximately $375 \mathrm{gm}$ were used as Ss.

Surgery. All rats underwent stereotaxic surgery under sodium pentobarbital anesthesia. Eight Ss received multiple, bilateral electrolytic lesions in the hippocampus. The electrode was not inserted into the brains of the six operated control Ss.

Training Apparatus and Procedures. One week after surgery Ss were placed on a 22-hr. food-deprivation schedule. Three weeks postoperatively four experimental and three control Ss began training on a 4-unit, multiple-T alley maze. At the same time, the other four experimental Ss and the other three control Ss were trained on an elevated $T$ maze of the same size and pattern. (See Hostetter \& Thomas, in press, for a more detailed description of the mazes.) After 30 trials (five consecutive trials per day for six days) Ss began training on the other maze. Thus, half of the experimental and control Ss learned first the alley maze and then the elevated maze, while the other half of the Ss learned the two mazes in reversed order. The Ss were allowed $30 \mathrm{sec}$. to eat wet mash in the goal box at the end of each trial. Guillotine doors in the stem of each $\mathrm{T}$ prevented retracing.

About 1 week after completion of the trials on the mazes, all Ss received a 4-min. open-field activity test in which the number of squares entered, the preferred location in the box (near the walls or in the center of the field), and the number of instances of defecation and urination were recorded.

Anatomical Findings. At the end of the experiment Ss with hippocampal lesions were perfused through the ascending aorta with physiological saline followed by $10 \%$ formalin. The brains were removed from the skulls, embedded in celloidin, and cut into $40-\mu$ thick frontal sections which were stained with cresyl violet and mounted. Drawings were traced from projected images of the sections, and the area of the lesion on each section was measured with a planimeter. The ratio of the sum of the lesioned area of hippocampus in the experimental brains to the total area of hippocampus on sections from a normal brain (X 100) yielded an approximate estimate of the percentage damage to the hippocampus.

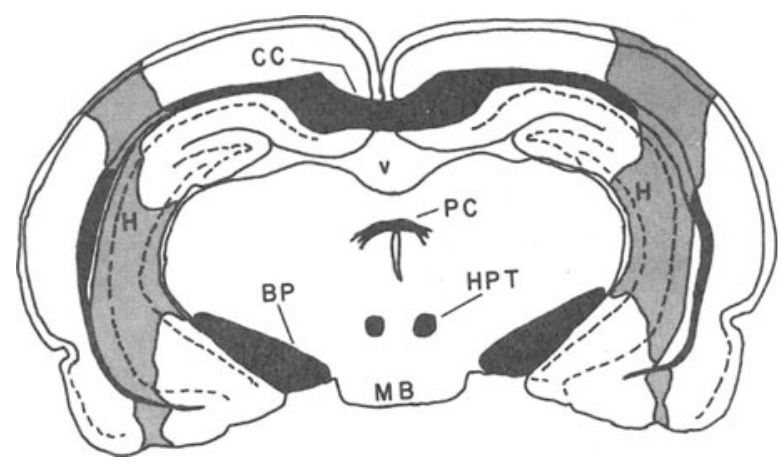

Fig. 1. Tracing of a frontal section through the maximal transverse extent of the lesions. Abbreviations: BP, basic peduncle; CC, corpus callosum; H, hippocampus; HPT, habenulo-peduncular tracts; MB, mamillary bodies; PC, posterior commissure; V, ventricle. 


\section{Results}

Anatomical Procedures. In all brains except one, the lesions were uniformly bilateral and were confined to the posterior and ventral portions of the hippocampus. The lesions were so similar in most of the brains that it was impossible to rank the brains reliably in terms of lesion size. In one brain the lesion was essentially unilateral. On the right side the lesion was extensive and involved neocortex and subcortical white matter. On the left side the lesion was confined to the hippocampus, but it was scarcely larger than an electrode track. Approximately $25 \%$ of the hippocampus in each brain (sum of damage to both sides) was destroyed. Extrahippocampal damage was limited. In no brain did the lesions invade the dorsal thalamus. In one brain the optic tract was unilaterally damaged. Figure 1 shows a drawing to illustrate typical brain damage. It was traced from a projected image of a section containing the largest transverse extent of the lesions.

Behavioral Findings. In the open-field test the experimental and control Ss did not differ significantly in number of squares entered or in the number of instances of urination and defecation. (Mann-Whitney U tests were used to evaluate statistically the data from the openfield test.) The hippocampal lesions certainly had no effect on emotionality or on spontaneous locomotor activity. The ratio of outer squares entered to the total number of squares entered in the open field was used as an index of wall-following tendency. The mean ratio for the experimental Ss was 0.88 , and the control Ss it was 0.73 . Both groups entered more squares near the walls (ratios between 0.5 and 1.0 ), but the difference between groups was not significant. The lesioned Ss do not show enhanced thigmotaxic tendencies in the open field.

Table 1 presents the mean number of errors in 30 trials for each treatment group on each maze for each order of maze learning. Comparison of the means in Table 1 shows that Ss with lesions in the hippocampus made more errors than control Ss on both the alley and elevated mazes. A 3-way analysis of variance (unweighted means solution with repeated measures design) ${ }^{1}$ indicated that the lesion effect was significant $(F=25.32, \mathrm{df}=1 / 10, \mathrm{p}<.01)$. The lesioned Ss made significantly more errors on both mazes than did control Ss. The maze effect was also significant $(F=$ 18.67 , df $=1 / 10, p<.01$ ). The treatment by maze interaction was not significant. Although both groups made fewer errors on the elevated maze than on the alley maze, the absence of a significant treatment by maze interaction contradicts the hypothesis that the performance of the experimental and control groups is differentially affected by the two types of maze. The effect of the third factor (order of learning) was not significant, nor was the order by maze interaction significant. It can be concluded that there was no sig-
Table 1. Mean Number of Errors in 30 Trials

\begin{tabular}{lccc} 
Group & $\mathrm{N}$ & Elevated & Alley \\
\hline & Alley learned before elevated & \\
Experimental & 4 & 47.0 & 64.8 \\
Control & 3 & 18.7 & 35.3 \\
& Elevated learned before alley & \\
Experimental & 4 & 75.3 & 86.8 \\
Control & 3 & 22.3 & 30.7 \\
\hline
\end{tabular}

nificant order effect and thus no significant transfer of training from the first to the second maze.

\section{Discussion}

The results of the maze-learning experiment agree with the findings of many other investigators that rats with hippocampal lesions are severely impaired in acquisition of a maze habit. Recently Gross, Chorover, \& Cohen (1965) suggested that large lesions that invade the lateral geniculate nuclei might account for the reported effects of "hippocampal lesions" on maze learning. The results of the present experiment do not support their hypothesis. The lesions in the hippocampus were all relatively small (approximately 25\%). In addition, none of the lesions extended into the lateral geniculate nuclei, and only one lesion extended into the optic tract. The lesioned rats, however, were significantly impaired in maze learning.

The results of this experiment do not illuminate the behavioral mechanisms by which lesions in the hippocampus interfere with maze performance. The lesions did not enhance thigmotaxic tendencies, although they did impair maze performance. The lesions were relatively small and did not invade the lateral geniculate nuclei, so the results do not support the view that large lesions in the hippocampus that invade the visual system are necessary to impair maze performance.

\section{References}

Gross, C. G., Chorover, S. L., \& Cohen, S. M. Caudate, cortical, hippocampal and dorsal thalamic lesions in rats: Alternation and Hebb-Williams maze performance. Neuropsychologia, 1965, 3, 53-68.

Hostetter, G., \& Thomas, G. J. Evaluation of enhanced thigmotaxis as a condition of impaired maze learning by rats with hippocampal lesions. J. comp. physiol. Psychol., in press.

Kimble, D. P. The effect of bilateral hippocampal lesions in rats. J. comp. physiol. Psychol., 1963, 56, 271-283.

\section{Notes}

1. This study was supported in part by Grant MH-01370 from the National Institute of Mental Health and in part by NSF Undergraduate Research Participation Program. The first author was an Undergraduate NSF Fellow in the Department of Psychology. This research was conducted in the laboratories of the Department of Physiology and Biophysics. The authors are indebted to Dr. A. Whimbey for advice on the statistical analysis and to Ruth $V$. Chalmers for preparing the histological material.

2. Now at the Brain Research Institute, University of California, Los Angeles.

3. Now at the Center for Brain Research, University of Rochester, New York. 